\title{
Singlet, triplet, electron and hole transport along single polymer chains Matthew Bird ${ }^{a^{*}}$, Gina Mauro ${ }^{\mathrm{a}}$, Lori Zaikowski ${ }^{\mathrm{a}, \mathrm{b}}$, Xiang Li ${ }^{\mathrm{a}}$, Obadiah Reid ${ }^{\mathrm{d}}$, Brianne Karten ${ }^{\mathrm{b}}$, Sadayuki Asaoka ${ }^{\mathrm{c}}$, Hung-Cheng Chen ${ }^{\mathrm{a}}$, Andrew R. Cook ${ }^{\mathrm{a}}$, Garry Rumbles ${ }^{\mathrm{d}}$ and John R. Miller ${ }^{\mathrm{a}^{*}}$ ${ }^{\mathrm{a}}$ Chemistry Department, Brookhaven National Laboratory, Upton, NY 11973 USA; ${ }^{\mathrm{b}}$ Chemistry and Physics Department, Dowling College, Oakdale, NY 11769 USA; ${ }^{\circ}$ Department of Biomolecular Engineering, Kyoto Institute of Technology, Matsugasaki, Sakyo-ku, Kyoto 606-8585, Japan; ${ }^{\mathrm{d}}$ National Renewable Energy Laboratory, Golden, Colorado 80401, United States
}

\begin{abstract}
The diffusion of singlet and triplet excitons along single polyfluorene chains in solution has been studied by monitoring their transport to end traps. Time-resolved transient absorption and steady state fluorescence were used to determine fractions of excitons that reach the end caps. In order to accurately determine the singlet diffusion coefficient, the fraction of polymer ends that have end traps was determined through a combination of NMR and triplet quenching experiments. The distributions of polymer lengths were also taken into account and the resulting analysis points to a surprisingly long singlet diffusion length of $34 \mathrm{~nm}$. Experiments on triplet transport also suggest that the entire 100nm+ chain is accessible to the triplet during its lifetime suggesting a lack of hindrance by defects or traps on this timescale. Time Resolved Microwave Conductivity measurements were also performed on a series of different length oligo- and polyfluorenes in solution allowing a global fit to be performed to extract an accurate intrachain mobility of $1.1 \mathrm{~cm}^{2} / \mathrm{Vs}$.
\end{abstract}

Keywords: Conjugated polymers, OPV, polyfluorene, microwave conductivity, exciton diffusion length, single chain

\section{INTRODUCTION}

Conjugated polymers have been studied extensively for use in devices such as photovoltaics, transistors and light emitting diodes. Typically such devices consist of films of polymers, or polymers blended with other molecules, and may exhibit various degrees of order. It is often difficult to decouple the intra- and inter-molecular transport as hopping from one chain to another is the process that usually limits transport over device-scale distances. Furthermore, the static disorder present in the solid state will tend to funnel charges and neutral excitations into lower energy sites, limiting their ability to transport over larger distances or equivalently limiting their mobility.

For organic photovoltaics, the bulk heterojunction has become the predominant device structure. The short diffusion lengths of 5-10 nm observed in polymer films mean that a compromised morphology is desired where fine phase separation that minimizes the distance to a donor-acceptor interface is balanced against better ordered large domains that give better charge transport out of the device. To see if a paradigm shift towards a more controlled structure where excitations and charges can be efficiently shuttled to their desired locations, over large distances, and with minimal energy loss, basic transport properties must be understood along single chains. This paper brings together a series of measurements on a model system of polyfluorene where charge, ${ }^{1}$ singlet ${ }^{2}$ and triplet $^{3}$ exciton transport has been studied. It is the unique set of polymers with well-defined lengths and end traps, ${ }^{4}$ as well as the use of techniques including pulse radiolysis and microwave conductivity that has enabled this work to be performed.

\section{EXPERIMENTAL}

\subsection{Materials}

The synthesis of the polyfluorenes $(\mathrm{pF})$ with end traps $(\mathrm{pFA})$, was described elsewhere. ${ }^{4}$ The polymers were separated by GPC into 9 fractions of different average lengths ranging from 20-118 repeat units, as determined by multi angle light scattering (MALS). The end traps were either anthraquinone (AQ) or naphthylimide (NI). The oligofluorenes had lengths ranging from $(\mathrm{n}=2-16)$. Benzene, $\mathrm{p}$-xylene, chloroform and tetrahydrofuran (THF) were used as received (Aldrich). Tetramethyl-p-phenylenediamine (TMPD) (Aldrich) was triply sublimed and biphenyl (Fisher Scientific) triply recrystallized prior to use.

*mbird@bnl.gov; jrmiller@bnl.gov 


\subsection{Triplet transport}

The Laser Electron Accelerator Facility ${ }^{5}$ (LEAF) at Brookhaven National Laboratory was used to generate $9 \mathrm{MeV} \sim 20 \mathrm{ps}$ electron pulses to make ionizations in p-Xylene leading to 1-4 $\mu \mathrm{M}$ of triplets. Solutions contained $100 \mathrm{mM}$ biphenyl to enhance the triplet yield and the polyfluorenes were at concentrations of 50-200 $\mu \mathrm{M}$ to ensure that no more than one triplet would be on a given chain. Transient absorption from the polymer triplets was measured using a pulsed Xenon lamp, $10 \mathrm{~nm}$ bandpass interference filters and a Silicon photodetector (FND 100).

\subsection{Singlet transport}

Fluorescence measurements were performed on 0.01 - $0.1 \mu \mathrm{M}$ polymer solutions in THF, chloroform and p-xylene using a Horiba Jobin-Yvon Fluoromax 4. Solution concentrations were adjusted to give the same absorbance at the excitation wavelength of $380 \mathrm{~nm}$. UV-VIS absorption spectra were taken with a Shimadzu 3600 spectrometer.

\subsection{Pulse-Radiolysis Time Resolved Microwave Conductivity (PR-TRMC)}

A Van de Graaff accelerator was used to produce $2 \mathrm{MeV} \sim 100$ ns electron pulses to ionize polymer solutions in benzene contained in a section of waveguide. Most ions undergo rapid geminate recombination to form excited states due to the low solvent polarity $(\varepsilon=2.28)$ but $\sim 10 \mathrm{nM}$ of charge pairs, that are formed at distances greater than the Onsager radius, last long enough to attach to solutes of $\sim 0.1-0.5 \mathrm{mM}$, before going on to decay by bimolecular recombination. To measure hole mobility, the solution is bubbled with oxygen (giving $\sim 10 \mathrm{mM}$ concentration) which captures electrons giving a negligible microwave conductivity. Microwave were generated with a Gunn diode at $8.9 \mathrm{GHz}$ and the reflected power from the cavity $(Q \sim 200)$ containing the solution was measured with a calibrated crystal detector. This reflection geometry with a resonant cavity for PR-TRMC measurements is described in more detail elsewhere. ${ }^{6,7}$

\section{RESULTS}

\subsection{Triplet transport}

To investigate triplet transport, pulse radiolysis was performed on solutions of the polymers in p-xylene, prepared under an inert atmosphere. Pulse radiolysis in non-polar solvents creates a large number of excited states and a relatively low yield of free ions. A large yield of triplets are formed in p-xylene alone from recombination of ions with appropriate spin combinations and intersystem crossing from singlet excited states $(\phi=0.63)$. However, the yield can be further enhanced by the addition of $100 \mathrm{mM}$ biphenyl which has a reported ${ }^{8}$ triplet yield of 0.84 and lifetime of $130 \mu \mathrm{s}$ which is easily sufficient to see the triplets live long enough to attach to the polymers that are at a lower concentration of $\sim 100 \mu \mathrm{M}$.

To determine the fraction of triplets that remain on the chain as a function of time, it is necessary for the triplets that reach the end trap to become trapped and have a distinguishable absorption spectrum. Previous experiments ${ }^{9}$ have shown that the triplets do get trapped on the end traps but they still have a significant optical absorption that is similar to that of the chain triplet. Due to the lower energy required to reduce the end trap compared to the chain $\left(\mathrm{pF}^{0 /-}=-2.65, \mathrm{pFNI}^{0 /-}=\right.$ 1.83, $\mathrm{pFAQ}^{0 /-}=-1.44$ all vs $\mathrm{Fc}^{+/ 0}$ ), it was found that the trapped triplet has sufficient energy to form a short-lived charge-transfer (CT) complex with TMPD whereas the chain triplet does not. Addition of TMPD to the solutions can convert trapped triplets into CT complexes $\left(\mathrm{pFA}^{\delta-}, \mathrm{TMPD}^{\delta+}\right.$ ) whose spectrum could be used to infer lifetimes of $240 \mathrm{~ns}$ and $1.13 \mu$ s for pFAQ and pFNI respectively. The absorption from the complexes peaks far from the pF triplet band making the $760 \mathrm{~nm}$ absorption a good indicator of the remaining chain triplet population.

Figure 1 shows transient absorption traces at 760nm from solutions containing pF, pFAQ and pFNI in THF with 10mM of TMPD and $100 \mathrm{mM}$ of biphenyl. From experiments with other TMPD concentrations, ${ }^{3}$ it was established that there is an equilibrium between the charge transfer complex and the end-trapped triplet with $\mathrm{K}_{\mathrm{eq}} \sim 4 \times 10^{4} \mathrm{M}^{-1}$. A concentration of $10 \mathrm{mM}$ of TMPD was sufficient to drive the equilibrium to be almost entirely (99.8\%) CT complex, essentially removing any absorption at $760 \mathrm{~nm}$ from triplets that reached the end trap. 


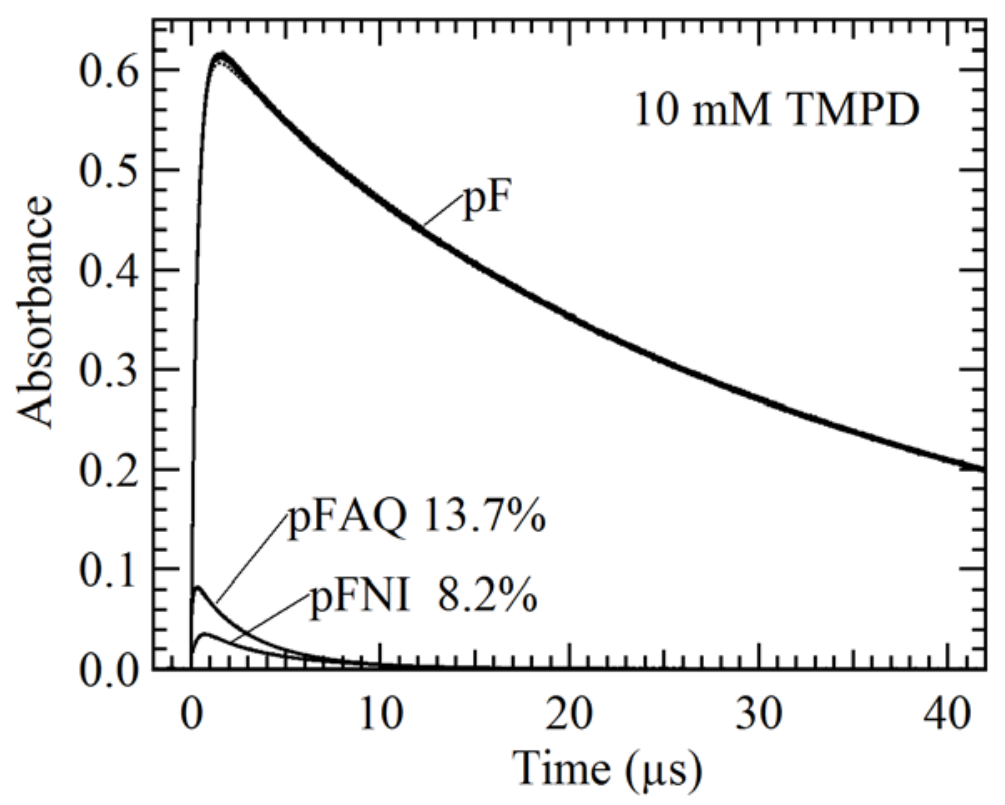

Figure 1. Triplet-triplet $\mathrm{pF}$ chain absorption at $760 \mathrm{~nm}$ following pulse radiolysis of solutions containing $\mathrm{pF}(100 \mu \mathrm{M})$, $\mathrm{pF}_{20} \mathrm{AQ}(200 \mu \mathrm{M})$ and $\mathrm{pF}_{22} \mathrm{NI}(100 \mu \mathrm{M})$ in THF with TMPD $(10 \mathrm{mM})$ and biphenyl $(100 \mathrm{mM})$. Adapted with permission from J. Phys. Chem. B, 2015, 119 (24), 7210. Copyright 2015 American Chemical Society.

As figure 1 shows, $13.7 \%$ and $8.2 \%$ of triplets remained on the chain for the $\mathrm{pF}_{20} \mathrm{AQ}$ and $\mathrm{pF}_{22} \mathrm{NI}$ polymers respectively. These remaining triplets decay at a rate which is proportional to the length of the chains; this is consistent with intermolecular transfer to other chains rather than intramolecular transport of the triplet to an end trap. The decay of the triplet absorption on the pFAQ and pFNI chains is therefore attributed to transfer to chains with at least one end trap, meaning that the percentages marked on figure 1 represent the number of chains with zero end traps.

For a series of different length polymers, the fraction of chains with no end traps, $F_{0}$, was used to determine the endcapping efficiency, $P_{\text {cap }}$, using:

$$
P_{\text {cap }}=1-\sqrt{F_{0}}
$$

The fraction of chains with one cap, $F_{1}$, and 2 caps, $F_{2}$, can then also be determined using:

$$
F_{1}=2 P_{\text {cap }}\left(1-P_{\text {cap }}\right), \quad F_{2}=P_{\text {cap }}^{2}
$$

The probability of an end being capped can also be found independently by NMR measurements ${ }^{3}$ although, for longer chains, the ratio of signal from the end traps to the chain becomes very large, reducing confidence in the estimated capping fraction. For short chains the NMR and triplet data agree well as shown in figure 2. 


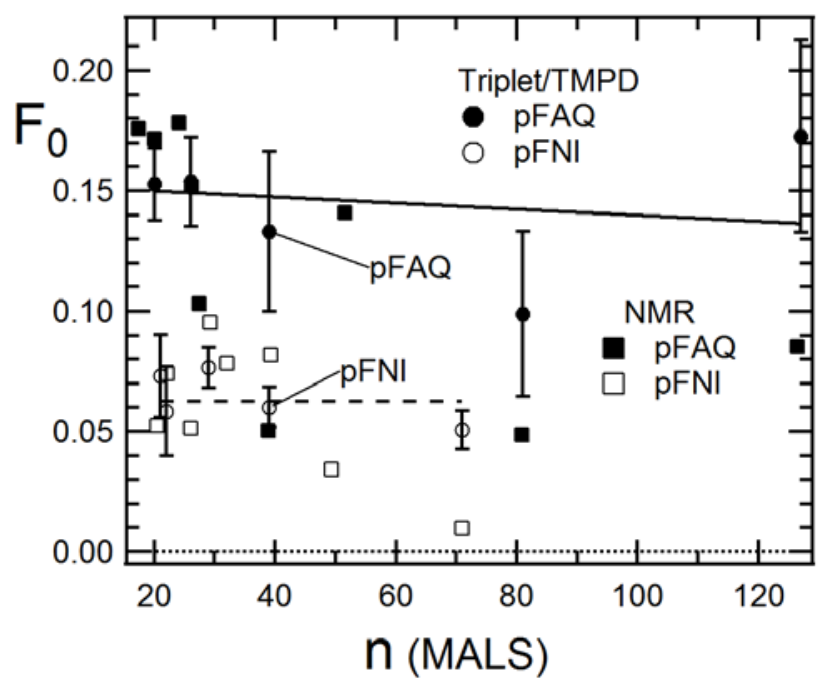

Figure 2. Fraction of pFAQ (filled markers) and pFNI (empty markers) chains with no end traps measured both with NMR (squares) and the triplet quenching experiment reported here (circles). Adapted with permission from J. Phys. Chem. B, 2015, 119 (24), 7210. Copyright 2015 American Chemical Society.

From averaging all the available data, the fraction of chains with no end traps were found to be 0.063 and 0.148 for pFAQ and pFNI respectively, giving the following fractions for zero, one and two end traps:

\begin{tabular}{ccc} 
& pFNI & pFAQ \\
\hline $\mathrm{F}_{0}$ & 0.06 & 0.15 \\
$\mathrm{~F}_{1}$ & 0.38 & 0.47 \\
$\mathrm{~F}_{2}$ & 0.56 & 0.38
\end{tabular}

While the time resolution is not yet sufficient to determine a diffusion coefficient for triplets, we can say that if there is at least one end trap on a chain, the triplet will find it during its lifetime, and in less than 40 ns. The result points to the single chains in solution allowing a continuous pathway for triplet transport. Secondly, we can determine the fraction of chain ends that were successfully capped in the synthesis which is vital for the analysis in the following section.

\subsection{Singlet transport}

Steady state fluorescence measurements with excitation at $380 \mathrm{~nm}$ were performed on absorption-matched solutions of polyfluorene chains of various lengths with and without end traps. As shown in figure 3 , the fluorescence from the chain gets quenched for chains with end traps and a new, red-shifted CT emission feature from the end-trapped excitons is observed. As would be expected, the data show that, as the chain length gets shorter, the chain fluorescence decreases and the CT emission concomitantly increases. The data therefore reflect the fact that transport along the chain competes with the $\tau=400$ ps measured radiative lifetime of the chain excitons and allows the diffusion coefficient, $D$, to be obtained. 


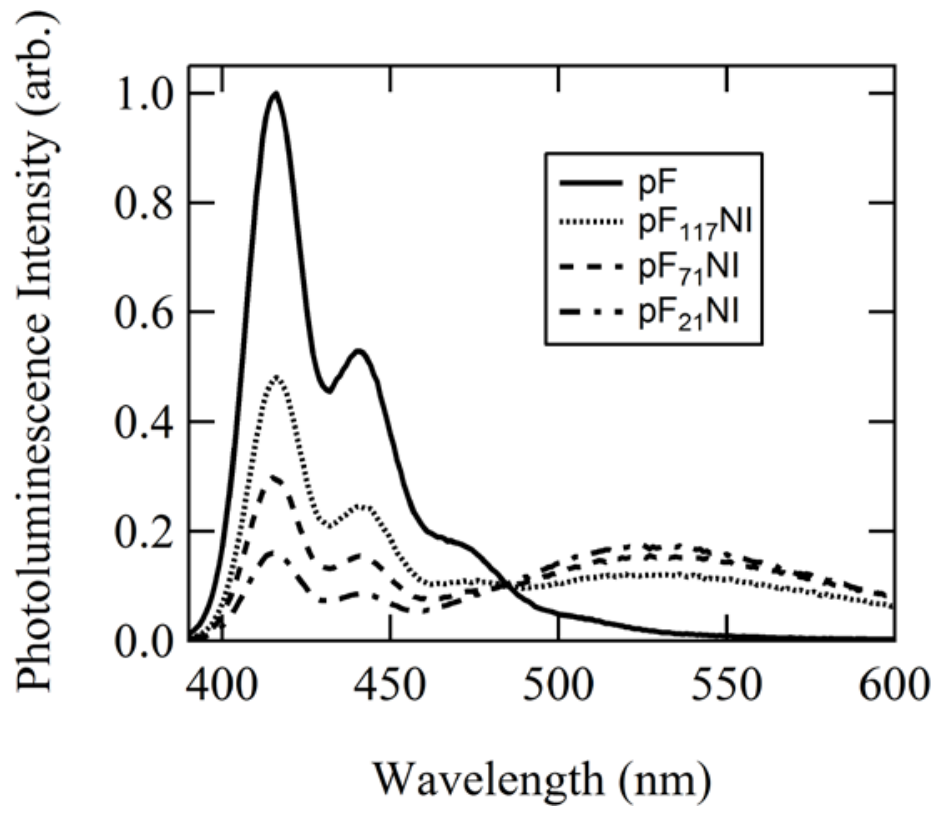

Figure 3. Fluorescence from absorption matched solutions of $\mathrm{pF}$ and $\mathrm{pFNI}$ of various lengths in THF showing the quenching of chain fluorescence at $415 \mathrm{~nm}$ and the rise of CT fluorescence from the NI end trap at $530 \mathrm{~nm}$. Adapted with permission from J. Phys. Chem. B, 2015, 119 (24), 7231. Copyright 2015 American Chemical Society.

A simple model of one-dimensional diffusion along a single chain with two sinks at the ends ${ }^{10,11}$ gives the following expression for the probability, $P(t)$, of an exciton still being on the chain at time, $t$ :

$$
P(t)=\frac{\sum_{n=0}^{\infty} \frac{1}{(2 n+1)^{2}} e^{-(2 n+1)^{2} \pi^{2} D t / L^{2}}}{\sum_{n=0}^{\infty} \frac{1}{(2 n+1)^{2}}}
$$

where $L$ is the length of the chain. For a chain with only one end trap $2 L$ would be used instead of $L$, under the assumption that the un-capped end on a singly-capped chain, would be indistinguishable from the center point of a chain of twice the length with end traps at both ends.

Knowing the fraction of chains with zero, one and two end traps from the previous section, a function was written, using equation 3 , to numerically calculate the fraction of remaining fluorescence from the chain, $F_{c}(L)$, relative to that from an uncapped chain:

$$
F_{c}(L)=\frac{1}{\tau} \int_{0}^{\infty}\left(F_{0}+F_{1} P_{2 L}(t)+F_{2} P_{L}(t)\right) e^{-\frac{t}{\tau}} d t
$$

The only unknown parameter in equation 4 is the diffusion coefficient, so a global fit of all the fluorescence data for the series of different length polymers was attempted to find $D$. As shown in figure 4 , a function that just uses the average length of the polymer batch cannot provide an adequate fit to the data. The square root dependence of root-mean-squared displacement on time means is would take four times longer to diffuse twice a given distance and is the likely reason why applying the function to a single average length does not accurately average the function over all the lengths in a given polymer distribution. 


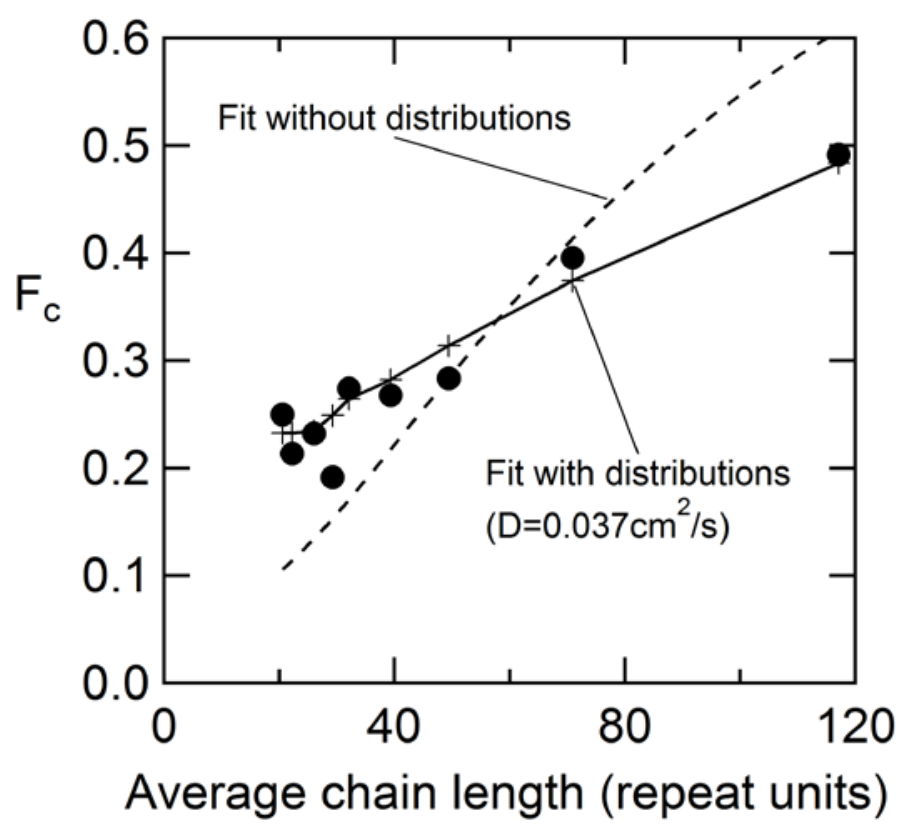

Figure 4. Chain fluorescence as a function of chain length for pFNI in THF $(\bullet)$ as a fraction of fluorescence from un-capped pF. Fits use one dimensional diffusion with $(+)$ and without $(--)$ averaging the function over the actual polymer length distributions. For the fit without distributions, the average chain length was used in the function. Adapted with permission from J. Phys. Chem. B, 2015, 119 (24), 7231. Copyright 2015 American Chemical Society.

Equation 4 was averaged over the measured polymer length distributions for each batch of polymers and again a global fit was performed to all the data. As shown in figure 4, taking the distribution of polymer lengths into account vastly improved the fit meaning that a simple diffusion model, used correctly, was able to reproduce the observed behavior of the excitons. The diffusion coefficient obtained from the data in figure 4, for the pFNI chains in THF, is $0.037 \mathrm{~cm}^{2} / \mathrm{s}$. In all, diffusion coefficients were found for the pFNI and pFAQ chains in THF, chloroform and xylene. The average diffusion coefficient from all six values was $0.029 \mathrm{~cm}^{2} / \mathrm{s}$, which coupled with the known exciton lifetime, gives a diffusion length, $L_{D}=\sqrt{D \tau}$ of $34 \mathrm{~nm}$, much larger than most reported values for polymers in films. ${ }^{12}$

\subsection{Hole transport}

The $9 \mathrm{GHz}$ microwave mobility of positive charges (holes) was measured for a series of lengths of oligo and polyfluorenes $(n=2-138)$ in benzene solutions using the PR-TRMC technique. The conductivity was calculated from the fractional change in reflected power, $\Delta P / P$, from the resonant cavity containing the polymer solution using the following equation:

$$
\frac{\Delta P}{P}=\mp \frac{Q\left(1 / \sqrt{R_{0}} \pm 1\right)}{\pi f_{0} \varepsilon_{0} \varepsilon_{r}} F \Delta \sigma
$$

where $Q$ is the quality factor of the cavity, $R_{0}$ is the fraction of power reflected by the cavity at resonance, $f_{0}$ is the resonance frequency, $F$ is the overlap factor between the electron-pulse induced charge density and the electric field and $\Delta \sigma$ is the change in conductivity in the region of the charge density; all other symbols have their standard meaning.

Figure 5 shows an example of a transient microwave conductivity trace from a solution of $\mathrm{pF}_{59}$ in oxygen-bubbled benzene following the electron pulse. The growth in conductivity corresponds to where the relatively low mobility benzene dimer cations transferring their charge to the polymer which gives a much large conductivity. The decay is due to bimolecular recombination of $\mathrm{pF}^{+}$with $\mathrm{O}_{2}^{-}$. 


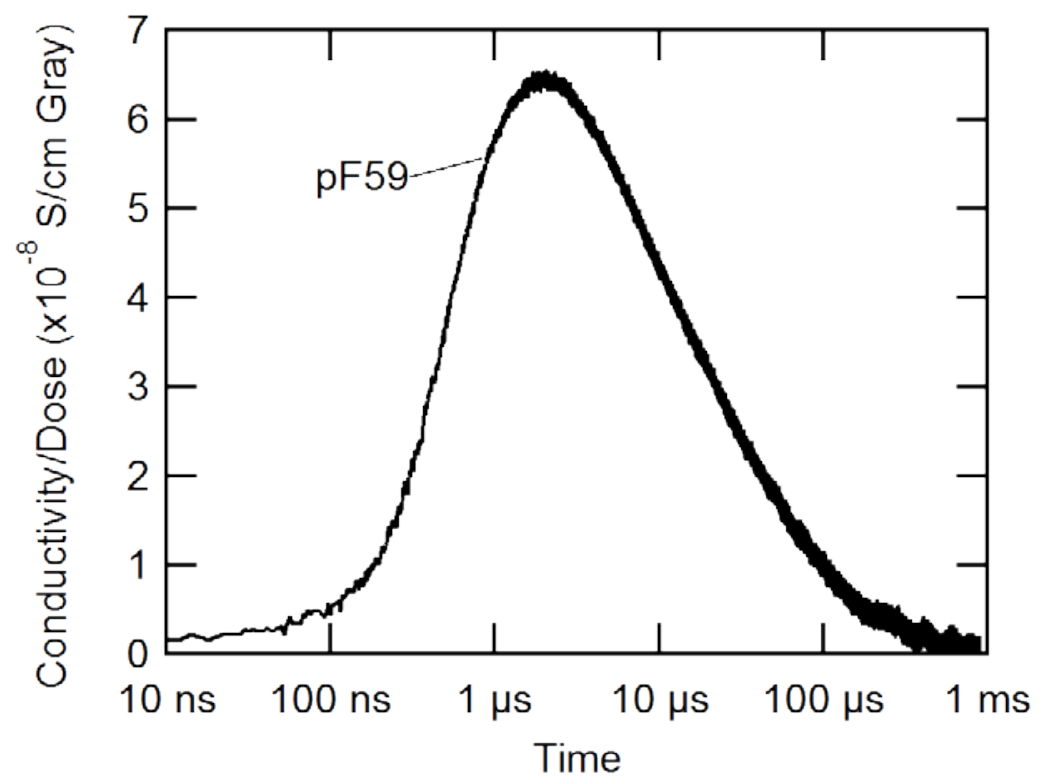

Figure 5. 8.9 GHz microwave conductivity per unit dose from a solution of pF59 in oxygen-bubbled benzene. Adapted with permission from J. Phys. Chem. C, 2014, 118 (12), 6100-6109. Copyright 2015 American Chemical Society.

The measured conductivity per unit dose, $\sigma_{D}$, was converted into a mobility using equation 6 by calculating the charge density per unit dose, $N_{D}$, in the solution by optical absorption measurements at $580 \mathrm{~nm}$ where the extinction coefficient of the $\mathrm{pF}$ cation is known.

$$
\sigma_{D}=N_{D} e \mu
$$

The solution contains polymers that are randomly orientated in three dimensions relative to the electric field which runs between the broad walls of the waveguide. The measured mobility is therefore often referred to as the isotropic mobility and would be $1 / 3$ of the mobility along the chain if the chains were straight and infinitely long. The situation becomes more complex however due to the fact that the chains have a finite length and are not straight. Figure 6 shows the measured isotropic mobility as a function of chain length for all the oligomers and polymers in this study. The general behavior can be understood in terms of a model developed by the Delft group. ${ }^{13}$ The standard mean squared displacement for diffusion in $n$ dimensional space, $\left\langle\Delta(t)^{2}\right\rangle=2 n D t$, can be inserted into the Kubo equation ${ }^{14}$ (7) to give the frequency response of a charged particle in the same system to an applied oscillating electric field of frequency $\omega$.

$$
\mu_{a c}=\frac{e \omega^{2}}{2 n k_{B} T} \int_{0}^{\infty}\left\langle\Delta(t)^{2}\right\rangle e^{-i \omega t} d t
$$

In this simple diffusion case, the standard Einstein relation is recovered, $\mu=D k_{B} T / e$. For a charge, confined on a polymer chain, the standard diffusional mean squared displacement is replaced with an analytical expression for $\left\langle\Delta(t)^{2}\right\rangle$ on a straight object with reflecting boundary conditions at the ends, averaged over all possible starting positions. The result is an analytical expression for the mobility, $\mu_{a c}^{1 D}$, for straight chains of length, $L$, aligned in the direction of the field: ${ }^{13}$ 


$$
\mu_{a c}^{1 D}=8 \mu_{\mathrm{int} r a} \sum_{k=0}^{\infty}\left[c_{k}^{2}\left(\frac{k_{B} T \mu_{\mathrm{int} r a} c_{k}^{2}}{i e \omega L^{2}}+1\right)\right]^{-1}
$$

where $c_{k}=2 \pi(k+1 / 2)$. Equation 8 assumes that if it were not for the end effects, normal diffusion would have occurred along the chain, giving $\mu_{\text {intra }}$ through the Einstein relation from $D$.

Figure 6 shows an attempt to fit equation 8 to the data where the function has been divided by three to account for the random orientation of polymer chains in space. The best fit gave an intramolecular mobility of $0.8 \mathrm{~cm}^{2} / \mathrm{Vs}$, but compared to the equation, the data appears to saturate at a lower mobility. Possible reasons for not reaching the theoretical maximum mobility are chain curvature, defects and non-monodisperse polymer length distributions.

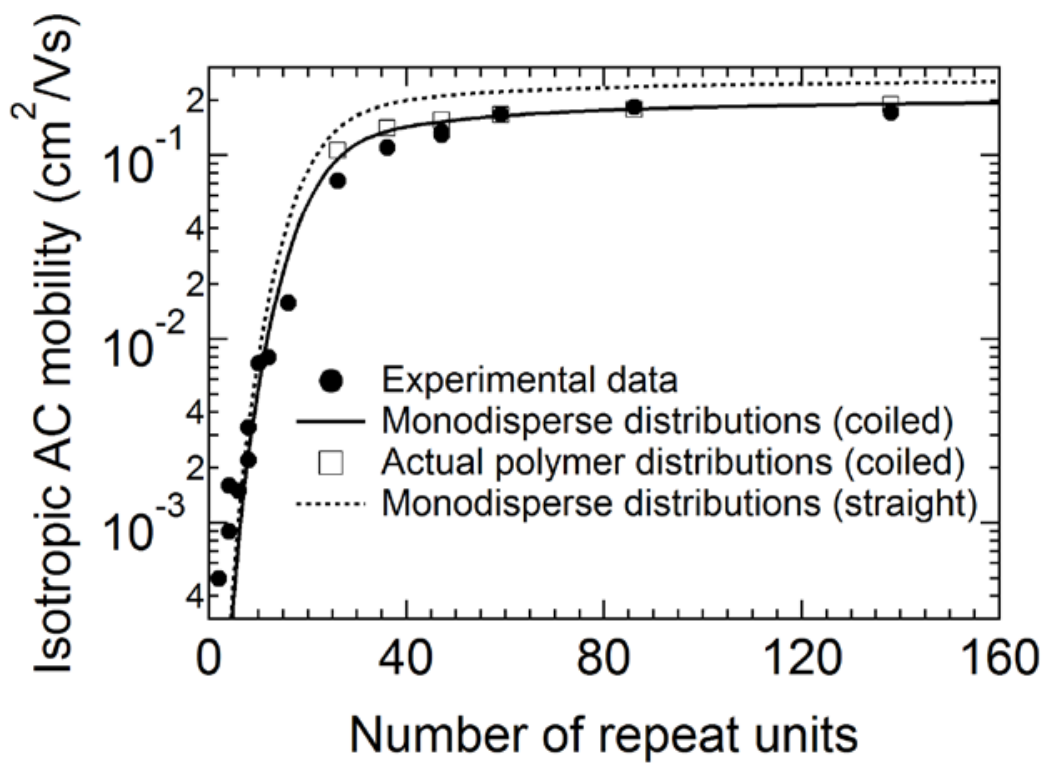

Figure 6. Measured isotropic AC mobility as a function of chain length for a series of oligo- and polyfluorenes. Dashed line represents a best fit using the model for straight chains. The solid line is the modified model that takes chain coiling into account and the open squares average this coiling model over the length distributions in each polymer batch. Adapted with permission from J. Phys. Chem. C, 2014, 118 (12), 6100-6109. Copyright 2015 American Chemical Society.

To incorporate, into the model, the fact that the chains are not straight, computer simulations of possible chain conformations, using the calculated dihedral angle potential (Gaussian $09^{15}$ DFT at the B3LYP/6-31g(d) level) and repeat unit length, were repeatedly made and the average relationship between the mean squared displacement along the contour of the chain to the mean squared displacement in space was found. The mean squared displacement in space as a function of time could then be computed, averaged over all starting positions and used in equation 7 to get a numerical solution to the isotropic AC mobility as a function of chain length as shown with the solid line in figure 6 . This method is essentially assuming normal diffusion along the contour of the chain as opposed to a more sophisticated method of computing the solution to the time-dependent Schrodinger equation for the evolution of the wave function of the charge. ${ }^{16}$ The best fit to the coiled model gives an intramolecular mobility of $1.1 \mathrm{~cm}^{2} / \mathrm{Vs}$. The function was also modified to average over all the lengths in a given polymer distribution and the values are shown as open squares in figure 6 revealing only a slight deviation from the monodisperse curve.

A similar fit to the data could be achieved with an alternative model of the chain being made up of relatively straight segments between kinks/defect which serve as barrier to charge transport. This defect model can also predict the premature saturating of mobility with chain length. For the best fit, the average defect spacing would have to be 16 repeat units, but this is longer than the persistence length of polyfluorene ${ }^{17}$ so cannot be a realistic model. On the other hand, combining the coiled model with the defect model gave a modest improvement in the fit and set a minimum spacing between defects of 30-40 repeat units. 


\section{DISCUSSION}

A surprising result from the data shown here, as summarized in table 1, is that the diffusion coefficients for singlet excitons and charges appear to be very similar despite the fact that energy and charge transport would be expected to proceed via different mechanisms. It is too early at this stage to know if this result is general. A recent report about barriers to transport along polyfluorene chains ${ }^{18}$ indicates that "bad" dihedral angles may serve as a higher barrier to singlet excitons than charges or triplet excitons. Despite this, it may be that there is some other factor that limits both kinds of transport but further experiments are required to address this question.

\begin{tabular}{lccc} 
& Singlet & Triplet & Charge \\
\hline Solvent & $\mathrm{THF} / \mathrm{CHCl}_{3} / \mathrm{p}$-xylene & $\mathrm{p}-\mathrm{xylene}$ & benzene \\
Diffusion Coefficient $\left(\mathrm{cm}^{2} / \mathrm{s}\right)$ & $2.9 \times 10^{-2}$ & $>3 \times 10^{-4^{*}}$ & $2.8 \times 10^{-2}$ \\
Diffusion Length & $34 \mathrm{~nm}$ & $>1 \mu \mathrm{m}$ & $\mathrm{n} / \mathrm{a}$
\end{tabular}

Table 1. Summary of diffusion coefficients for the various species along polyfluorene chains. $*$ Taken from ref ${ }^{9}$

At present, only a lower limit for the diffusion coefficient of triplets along polyfluorene chains has been found. Efforts are ongoing to improve the time resolution of the triplet transport experiment to find out how it compares.

\section{CONCLUSION}

Transport of excitons and charges were studied on a series of length-separated polyfluorenes using steady state fluorescence, pulse radiolysis and microwave conductivity. Triplets, generated by pulse radiolysis, attached onto single polymer chains in xylene and, if at least one end trap was present, would reach it within the time resolution of the experiment. The conclusion from the triplet data is that the chains allow a continuous path for triplets on the $\mu$ s time scale which also allowed the fraction of successful end capping by trap groups to be determined. Steady state fluorescence measurements were taken for the same set of end-capped polymers and was modelled with a simple diffusion model. When taking into account the correct end capping fraction and length distributions, a good fit could be obtained to complete datasets giving an average diffusion coefficient of $0.029 \mathrm{~cm}^{2} / \mathrm{s}$ which corresponds to an unprecedented diffusion length of $34 \mathrm{~nm}$. Finally, microwave conductivity measurements were performed for the same set of length-separated polyfluorenes in benzene, enabling a global fit to be performed which suggests an intrinsic, along chain, mobility of $1.1 \mathrm{~cm}^{2} / \mathrm{Vs}$. In all cases, no strong evidence of permanent barriers to transport along the chain were found.

\section{ACKNOWLEGEMENTS}

The authors gratefully acknowledge support of the Solar Photochemistry Program, Division of Chemical Sciences, Geosciences, and Biosciences, Office of Basic Energy Sciences of the U.S. Department of Energy, under contract numbers DE-AC02-98-CH10886 and DE-SC0012704 (BNL, including use of the LEAF and Van de Graaff facilities of the BNL Accelerator Center for Energy Research) and DE-AC36-08GO28308 (NREL) for polymer research. Work of M.B. and O.R. to develop the Pulse-Radiolysis TRMC experiment was supported by Laboratory Directed Research Grants 02544 at BNL and 06RF1002 at NREL. Partial support was provided by the National Science Foundation's Robert Noyce teacher scholarship Program at Dowling College to L.Z., B.K. and G.M. under Award No. 03-35799 and 09-34814. L.Z. and G.M. thank Noel Blackburn of the BNL Office of Educational Programs for his leadership and support in the U.S. Department of Energy Faculty and Student Team (FaST) Program. L.Z. acknowledges support from Dowling College Release Time for Research. 


\section{REFERENCES}

[1] Bird, M. J., Reid, O. G., Cook, A. R., Asaoka, S., Shibano, Y., Imahori, H., Rumbles, G. and Miller, J. R., "Mobility of Holes in Oligo- and Polyfluorenes of Defined Lengths," The Journal of Physical Chemistry C, 118, 6100 (2014)

[2] Zaikowski, L., Mauro, G., Bird, M., Karten, B., Asaoka, S., Wu, Q., Cook, A. R. and Miller, J. R., "Charge Transfer Fluorescence and $34 \mathrm{~nm}$ Exciton Diffusion Length in Polymers with Electron Acceptor End Traps," The Journal of Physical Chemistry B, 119, 7231 (2014)

[3] Li, X., Bird, M., Mauro, G., Asaoka, S., Cook, A. R., Chen, H.-C. and Miller, J. R., "Transport of Triplet Excitons along Continuous $100 \mathrm{~nm}$ Polyfluorene Chains," The Journal of Physical Chemistry B, 119, 7210 (2015)

[4] Asaoka, S., Takeda, N., Lyoda, T., Cook, A. R. and Miller, J. R., "Electron and hole transport to trap groups at the ends of conjugated polyfluorenes," Journal of the American Chemical Society, 130, 11912 (2008)

[5] Wishart, J. F., Cook, A. R. and Miller, J. R., "The LEAF picosecond pulse radiolysis facility at Brookhaven National Laboratory," Review of Scientific Instruments, 75, 4359 (2004)

[6] Infelta, P. P., de Haas, M. P. and Warman, J. M., "The study of the transient conductivity of pulse irradiated dielectric liquids on a nanosecond timescale using microwaves," Radiation Physics and Chemistry, 10, 353 (1977)

[7] Warman, J. M., Infelta, P. P., De Haas, M. P. and Hummel, A., "The study of primary and secondary charge carriers in nanosecond pulse irradiated liquid dielectrics using a resonant microwave cavity," Canadian Journal of Chemistry, 55, 2249 (1977)

[8] Murov, S. L., Carmichael, I. and Hug, G. L., [Handbook of Photochemistry, 2nd ed.], Marcel Dekker: New York (1993)

[9] Sreearunothai, P., Estrada, A., Asaoka, S., Kowalczyk, M., Jang, S., Cook, A. R., Preses, J. M. and Miller, J. R., "Triplet Transport to and Trapping by Acceptor End Groups on Conjugated Polyfluorene Chains," Journal of Physical Chemistry C, 115, 19569 (2011)

[10] Zachmanoglou, E. C. and Thoe, D. W., [Introduction to Partial Differential Equations with Applications], Dover Publications (1987)

[11] Carslaw, H. S. and Jaeger, J. C., [Conduction of Heat in Solids, 2nd ed.], Oxford University Press (1986)

[12] Mikhnenko, O. V., Blom, P. W. M. and Nguyen, T.-Q., "Exciton diffusion in organic semiconductors," Energy \& Environmental Science, 8, 1867 (2015)

[13] Prins, P., Grozema, F. C., Schins, J. M. and Siebbeles, L. D. A., "Frequency dependent mobility of charge carriers along polymer chains with finite length," Physical Status Solidi B, 243, 382 (2006)

[14] Kubo, R., "Statistical-Mechanical Theory of Irreversible Processes. I. General Theory and Simple Applications to Magnetic and Conduction Problems," Journal of the Physical Society of Japan, 12, 570 (1957)

[15] Frisch, M. J., Trucks, G. W., Schlegel, H. B., et al. Gaussian 09, Gaussian, Inc.: Wallingford CT (2009).

[16] Prins, P., Grozema, F. C., Galbrecht, F., Scherf, U. and Siebbeles, L. D. A., "Charge transport along coiled conjugated polymer chains," Journal of Physical Chemistry C, 111, 11104 (2007)

[17] Grell, M., Bradley, D. D. C., Long, X., Chamberlain, T., Inbasekaran, M., Woo, E. P. and Soliman, M., "Chain geometry, solution aggregation and enhanced dichroism in the liquidcrystalline conjugated polymer poly(9,9dioctylfluorene)," Acta Polymerica, 49, 439 (1998)

[18] Mani, T. and Miller, J. R., "Role of Bad Dihedral Angles: Methylfluorenes Act as Energy Barriers for Excitons and Polarons of Oligofluorenes," The Journal of Physical Chemistry A, 118, 9451 (2014) 